\title{
Undiagnosed Abdominal Paraganglioma: An Anaesthetic Challenge $^{*}$
}

\section{Lalit Gupta\#, Sanjay Kr. Nihalani, Saipriya Tiwari, Poonam Bhadoria, Sonia Wadhawan}

Department of Anaesthesia \& Intensive Care, Maulana Azad Medical College \& Associated Lok Nayak Hospital, New Delhi, India; Email: "1alit.doc@gmail.com

Received October $21^{\text {st }}, 2013$; revised November $22^{\text {nd }}, 2013$; accepted December $3^{\text {rd }}, 2013$

Copyright (C) 2013 Lalit Gupta et al. This is an open access article distributed under the Creative Commons Attribution License, which permits unrestricted use, distribution, and reproduction in any medium, provided the original work is properly cited.

\begin{abstract}
Paragangliomas are tumours which arise in extra adrenal autonomic paraganglia and have the ability to secrete catecholamines. Their anaesthetic management is the same as that of a case of pheochromocytoma, but in an undiagnosed case like our patient, unexpected eventful encounter in the operating theatre may lead to life threatening complications during surgical resection warranting immediate management and thus posing a real challenge to the skill of an anaesthesiologist.
\end{abstract}

Keywords: Abdominal Paraganglioma; Ventricular Tachycardia; Hypertensive Crisis

\section{Introduction}

We report a case of a 75-year-old patient with an intra abdominal swelling provisionally diagnosed as a retroperitoneal tumour and posted for tumour resection. The patient suffered an intraoperative hypertensive crisis, ventricular tachycardia and hypotension which were successfully managed.

\section{Case Report}

A 75-year-old male patient of Indio-Asian origin with an intra abdominal swelling provisionally diagnosed as retroperitoneal tumour was posted for laparotomy and resection. The patient had no significant medical history and all his investigations were normal except hemoglobin of $9.4 \mathrm{gm} \%$.

CECT scan abdomen showed a large solid cystic mass, extending from the upper pole of the right kidney to the level of the pubic symphysis. Provisional diagnosis of a large Retroperitoneal mass or Mesothelioma was made by radiologist.

Patient was transfused 1 unit packed cells preoperatively and was taken up for surgery under general anaesthesia with epidural analgesia. All routine monitors were attached and baseline parameters recorded (heart rate

\footnotetext{
*Competing interests: The authors declare that they have no competing interests.

${ }^{\#}$ Corresponding author.
}

60/min, BP124/78 mm Hg). 19G epidural catheter placed in $\mathrm{L}_{2}-\mathrm{L}_{3}$ space and correct placement verified by test dose (45 mg of lignocaine with $15 \mu \mathrm{g}$ of epinephrine).

The patient was premedicated with Fentanyl $100 \mu \mathrm{g}$ and Midazolam $1 \mathrm{mg} \mathrm{I/V}$. Anaesthesia was induced with Propofol $2 \mathrm{mg} / \mathrm{kg}$, Rocuronium bromide $1 \mathrm{mg} / \mathrm{kg} \mathrm{I} / \mathrm{V}$, Isoflurane $(0.6 \%)$, Nitrous oxide and oxygen. After ventilating the patient for 3 minutes, orotracheal intubation was done. Anaesthesia was maintained with isoflurane $(0.6 \%-1 \%)$, nitrous oxide and oxygen (2:1) and muscle relaxation maintained with Rocuronium bromide I/V. Surgical incision was given and exploratory laparotomy was started. Initially for the first 20 minutes, the vitals remained stable. However, during manipulation of the retroperitoneal mass, ventricular ectopics were seen on ECG with a frequency of more than $5 / \mathrm{min}$ with a heart rate of $72 / \mathrm{min}$. Xylocard $1.5 \mathrm{mg} / \mathrm{kg} \mathrm{I} / \mathrm{V}$ was given but the ECG rhythm changed to ventricular bigeminy within 2 minutes and blood pressure raised to $170 / 100 \mathrm{~mm} \mathrm{HG}$. Xylocard $0.75 \mathrm{mg} / \mathrm{kg}$ was repeated and isoflurane increased to $1.5 \%$. Radial pulses were palpable throughout.Soon after the rhythm changed to Ventricular tachycardia. Peripheral pulses were present and blood pressure further shot up to $210 / 114 \mathrm{~mm} \mathrm{Hg}$. We asked the surgeon to stop manipulating the mass. Amiodarone 150 $\mathrm{mg}$ slow $\mathrm{I} / \mathrm{V}$ was given followed by amiodarone infusion (a) $60 \mathrm{mg} / \mathrm{hr}$. Meanwhile, surgery was stopped, defibrillator was kept as standby, radial pulse was continuously 
monitored and NTG infusion was prepared. The patient had stable ventricular tachycardia for 10 minutes and after starting amiodarone infusion, ventricular tachycardia reverted back to sinus rhythm with a heart rate of 74/min and blood pressure 140/100 mm Hg. CVP line was secured.

The surgeon resumed the operation with the aim to proceed only if the mass was easily resectable otherwise to abandon the surgery. Graded epidural with $1 \%$ Xylocard was given up to a dose of $10 \mathrm{ml}$ over 10 minutes. After thirty minutes of starting amiodarone infusion, a large tumour mass measuring $20 \times 15 \mathrm{~cm}$ was dissected out of the abdomen. Blood pressure suddenly plummeted to $80 / 40 \mathrm{~mm} \mathrm{Hg}$ after resection of tumour. Amiodarone infusion was stopped. $500 \mathrm{ml}$ hydroxyethyl starch and 1 Lt. Ringer-Lactate were pushed I/V fast. Further fluid replacement was guided by CVP. Blood pressure increased to $90 / 60 \mathrm{~mm} \mathrm{Hg}$ and heart rate was maintained around $84 / \mathrm{min}$. Intraoperative blood loss was around 400 $\mathrm{ml}$. 1 unit packed cell was infused I/V and blood pressure stabilized to $110 / 70 \mathrm{mmHg}$. Intraoperatively, ArterialBloodGas (ABG) analysis was done. It showed $\left(\mathrm{FiO}_{2}\right.$ 0.40) $\mathrm{pH} 7.28 / \mathrm{pCO}_{2} 44 \mathrm{mmHg} / \mathrm{pO}_{2} 212 \mathrm{mmHg} / \mathrm{HCO}_{3}$ $20.3 \mathrm{mmol} / \mathrm{L} / \mathrm{B} . \mathrm{E}-6 \mathrm{mmol} / \mathrm{L} / \mathrm{SaO}_{2} 99.6 \%$.

Rest of the intraoperative period was uneventful. After completion of surgery, Anaesthesia was reversed with Glycopyrolate $10 \mu \mathrm{g} / \mathrm{kg}$ and Neostigmine $0.05 \mathrm{mg} / \mathrm{kg}$ IV and the patient's trachea was extubated. The patient was conscious, oriented with stable hemodynamics, maintaining adequate oxygen saturation of $97 \%-98 \%$ with Oxygen via ventimask.

Patient was shifted to the ICU for observation. Inj. Morphine $2.5 \mathrm{mg}$ diluted in $8 \mathrm{ml}$ saline was given epidurally in the ICU. Postoperatively, 12 lead ECG and cardiac isoenzymes were done. His blood pressure, pulse rate and oxygen saturation remained within normal limits and no other episode of arrhythmia occurred. Patient was shifted to the ward after $36 \mathrm{hrs}$ observation in ICU.

Later, histopathology report confirmed the tumour as paraganglioma of abdomen with concurrent tuberculosis.

\section{Discussion}

Paragangliomas are tumours which arise in extra adrenal sympathetic paraganglia. $20 \%$ of pheochromocytomas are extra adrenal in location, with the majority being located in the abdomen in association with the sympathetic ganglia $[1,2]$. Patients with paraganglioma usually present with sweating, headache, palpitations and pallor [3]. Hypertension sustained or paroxysmal has been reported in $80 \%-100 \%$ of patients [4].

Paragangliomas are rather seldom, mainly between the ages of 30 - 60 years, with no significant sex predisposi- tion [5]. The unexpected encounter of paraganglioma in the operating theatres, delivery rooms and other circumstances carries a mortality rate of $25 \%-50 \%$ [1].

Paragangliomas release an excessive amount of catecholamines (norepinephrine, epinephrine, dopamine) and metabolites (metanephrine and vanillylmandelic acid). At present, most centres diagnose a paraganglioma by urine testing for free catecholamines and their metabolites and/ or measuring plasma catecholamines.

In patients with raised serum catecholamine concentrations, preoperative administration of alpha adrenergic blocker-phenoxybenzamine, prazosin or phentolamine may be done. The major goal is to partially block the responses to catecholamines although surgery remains the only definitive therapy [6].

An asymptomatic abdominal paraganglioma is almost impossible to be diagnosed preoperatively, because the only guiding information is the clinical signs and symptoms derived from catecholamine release. In our patient, nothing was suggestive of catecholamine secreting mass preoperatively. So, no preparation was done on these lines thus leading to life threatening cardiovascular complications. It has been reported that despite active metabolites being secreted, paraganglioma cases may remain normotensive [7].

During surgery when hypertension and arrhythmia occurred, we suspected the mass to be a catecholamine secreting tumour after excluding light plane of anaesthesia, hypoxemia, hypercarbia, inadequate analgesia and acted promptly. The biopsy report of the resected mass confirmed it to be paraganglioma of the abdomen. In our patient, timely intervention during surgery by increasing the depth of anaesthesia, ensuring adequate analgesia, use of anti-arrhythmics-lidocaine and amiodarone, stopping of surgery, skillful surgical assistance and adequate maintenance of intravascular volume all helped to save the patient.

To conclude, any patient with a retroperitoneal tumour even without endocrinological symptoms should be suspected to be a paraganglioma and further investigations are warranted preoperatively before administering anaesthesia. It is important to have in mind the possible presence of this which is usually clinically silent but still hormonally active tumor when sudden unexplained hemodynamic instability occurs intra-operatively. A thorough differential diagnosis is essential in order to plan the best treatment and therefore gain the best outcome for the patient.

\section{Acknowledgements}

We are grateful to our patient, who agreed to the publication of this case report and provided all information needed. 


\section{REFERENCES}

[1] R. L. Hines and K. Marschall, "Stoelting's Anaesthesia and Co-Existing Disease," 5th Edition, Churchill Livingstone, Philadelphia, 2008, pp. 388-393.

[2] N. Kimura, C. Capella, R. R. De Krijger, L. D. R. Thompson, K. Y. Lam, P. Komminoth, A. S. Tischler and W. F. Young, "Extra-Adrenal Symphathetic Paraganglioma: Superior and Inferior Paraaortic," In: R. A. DeLellis, R. V. Lloyd, P. U. Heitz and C. Eng, Eds., World Health Organization Classification of TumoursTumours of Endocrine Organs, IARC Press, Lyon, 2004, pp. 164-165.

[3] S. G. Sheps, N. S. Jiang, G. G. Klee, et al., "Recent developments in the diagnosis and treatment of pheochromocytoma," Mayo Clinic Proceedings, Vol. 65, No. 1, 1990 , pp. 88-95.

http://dx.doi.org/10.1016/S0025-6196(12)62113-2
[4] M. T. Sanjoaquin, J. R. Fraile, A. Gutierrez, et al., "Hypertensive Crisis during a Transurethral Resection of the Bladder: Nondiagnosed Bladder Paraganglioma-A Case Report," Anesthesia \& Analgesia, Vol. 90, No. 3, 2000, pp. 556-558. http://dx.doi.org/10.1097/00000539-200003000-00011

[5] M. Gmeiner and J. Pfeifer, "Extraadrenal Abdominal Paraganglia: A Therapeutical Challenge," The Internet Journal of Surgery, Vol. 5, No. 1, 2004, p. 21.

[6] K. G. E. Pheochromocytoma, "Yao and Artusio's Anesthesiology: Problem-Oriented Patient Management," 5th Edition, Lippincott Williams \& Wilkins, Lippincott, 2004, p. 716 .

[7] C. K. Pandey, "Life Threatening Complications during Surgery in a Patient with Undiagnosed Abdominal Paraganglioma-A Case Report," Indian Journal of Anaesthesia, Vol. 44, 2000, pp. 54-56. 